\title{
ESTATUS PONDERAL Y APTITUD CARDIORRESPIRATORIA EN ESCOLARES DE LA REGIÓN CENTRAL DEL PERÚ
}

\author{
Alcibíades Bustamante ${ }^{1,2, a}$, José Maia $2, \mathrm{~b}$
}

RESUMEN

Objetivos. Determinar la frecuencia del sobrepeso y obesidad, en función a los niveles de aptitud cardiorrespiratoria (AptCR) en escolares de cuatro distritos de la región central del Perú, y analizar la asociación entre estas variables. Materiales y métodos. El peso, altura y la AptCR fueron evaluados en 7841 escolares que residen en cuatro distritos ubicados en la costa, sierra y selva de la región central del Perú. El sobrepeso y la obesidad fueron clasificados según los criterios propuestos por Cole. La AptCR fue evaluada mediante la prueba de correr/caminar de doce minutos de la batería de la American Alliance for Health, Recreation and Dance. Se utilizó el ANOVA y la regresión logística para examinar las diferencias de las medias y asociaciones entre estas variables. Resultados. Escolares de ambos sexos presentan frecuencias semejantes de sobrepeso y obesidad (20,9\% en mujeres y $20,1 \%$ en varones). Residentes en la costa (Barranco) manifiestan elevadas frecuencias de sobrepeso y obesidad (37,8\%). La edad, el sexo, área geográfica y la AptCR fueron predictores significativos para el sobrepeso y la obesidad. Escolares que viven en Barranco tienen cinco veces más probabilidades de ser obesos (OR=4,67; IC95\%: 3,55-6,14); los que residen en la sierra (Junín) tienen una menor probabilidad de ser obesos (OR=0,03; IC95\%: 0,01-0,20); y quienes muestran una baja AptCR tienen una mayor probabilidad de ser obesos (OR=11,82; IC95\%: 7,25-19,27), en comparación con aquellos con alta AptCR. Conclusiones. Los escolares que residen en Barranco presentan frecuencias de sobrepeso y obesidad elevados. Una baja AptCR está asociada al desarrollo del sobrepeso y la obesidad.

Palabras clave: Sobrepeso; Obesidad; Fenómenos fisiológicos circulatorios y respiratorios; Adolescente (fuente: DeCS BIREME).

\section{WEIGHT STATUS AND CARDIORESPIRATORY FITNESS IN SCHOOL STUDENTS IN THE CENTRAL REGION OF PERU}

\section{ABSTRACT}

Objectives. To determine the frequency of overweight and obesity in relation to cardiorespiratory fitness (CRF) levels in school students in 4 districts of the central region of Peru, and to analyze the relations among these variables. Materials and methods. Weight, height and CRF were evaluated in 7841 school students who reside in four districts located on the coast, in the highlands and in the jungle of the central region of the country. Overweight and obesity were classified according to the criteria proposed by Cole. CRF was evaluated by a 12-minute run/walk test taken from the American Alliance for Health, Physical Education, Recreation and Dance's test battery. ANOVA and logistic regression were used to examine the differences of the averages and the associations among these variables. Results. Both male and female school students have similar frequency of overweight and obesity (20.9\% in women and $20.1 \%$ in men). Residents of the coast (Barranco) presen thigh frequency of overweight and obesity (37.8\%). Age, sex, geographical area and CRF were significant predictors of overweight and obesity. School students who live in Barranco are five times more likely to be obese (OR=4.67; Cl95\%: 3.55-6.14), while those who reside in the highlands (Junin) are less likely to be obese $(\mathrm{OR}=0.03 ; \mathrm{Cl} 95 \%$ : 0.01-0.20). Furthermore, in contrast with students with high $\mathrm{CRF}$, those with low CRF are more likely to be obese (OR=11.82; Cl95\%: 7.25-19.27). Conclusions. There was a high frequency of overweight and obesity among school students who reside in Barranco. Low CRF is associated with overweight and obesity.

Key words: Overweight; Obesity; Circulatory and respiratory physiological phenomena; Adolescent (source: MeSH NLM).

\section{INTRODUCCIÓN}

En el 2010 la Organización Mundial de la Salud (OMS) (1) señaló que cerca de 40 millones de niños menores de 5 años presentaban sobrepeso. Con lo cual, la obesidad se convirtió en el quinto factor de riesgo de muerte en el mundo, representando una amenaza creciente para la salud tanto en países desarrollados como en vías de desarrollo ${ }^{(2)}$.

La Asociación Internacional para el Estudio de la Obesidad (3) señala que las prevalencias según sexo de sobrepeso y obesidad para menores de 5 a 17 años en Argentina son de 27,5 y 32,1\%; en Chile, de 27,1 y

\footnotetext{
Universidad Nacional de Educación Enrique Guzmán y Valle, Lima, Perú.

Centro de Investigação, Formação, Intervenção e Inovação em Desporto (CIFI²D), Faculdade de Desporto da Universidade do Porto. Porto, Portugal.

Magíster en Desarrollo Motor; ${ }^{\mathrm{b}}$ Doutor em Ciências do Desporto

Recibido: 21-04-13 Aprobado: 17-07-13
}

Citar como: Bustamante A, Maia J. Estatus ponderal y aptitud cardiorrespiratoria en escolares de la región central del Perú. Rev Peru Med Exp Salud Publica. 2013;30(3): 399-407. 
$28,6 \%$; en Brasil, de 23,1 y $21,1 \%$, y en Venezuela, de 22,4 y $14,8 \%$, para varones y mujeres respectivamente. Por otro lado, en Ecuador, un estudio en adolescentes entre 12 y 19 años de edad mostró una prevalencia de sobrepeso y obesidad del $21,5 \%$ en mujeres y $20,8 \%$ en varones ${ }^{(4)}$. En el Perú, a partir de información recogida en la Encuesta Nacional de Hogares (ENAHO, 2009-2010), se estimó que niños menores de 5 años, de 5 a 9 años y adolescentes entre 10 y 19 años presentan un 8,$2 ; 24,4$ y $14,2 \%$ de sobrepeso y obesidad, respectivamente ${ }^{(5)}$.

El aumento de la obesidad en las últimas décadas ha sido explicado, genéricamente, por múltiples y complejas interacciones entre genes y diversos factores ambientales ${ }^{(6)}$. Los cambios en los hábitos dietéticos, sobre todo el incremento del consumo de alimentos muy densos calóricamente y la adopción de estilos de vida sedentario que se manifiesta en bajos niveles de actividad y aptitud física durante la niñez y adolescencia pueden conducir a diversas complicaciones de salud en la edad adulta, como la diabetes, la hipertensión, la intolerancia a la glucosa, una función pulmonar más pobre y una amplia gama de condiciones patológicas menos comunes que, a su vez, pueden conllevar a condiciones de salud crónicas ${ }^{(7,8)}$.

La aptitud física expresa la interacción e integración de funciones y estructuras corporales ${ }^{(9)}$, y su componente cardiorrespiratoria es considerada como un predictor del estado de salud y bienestar de las personas ${ }^{(10)}$. La aptitud cardiorrespiratoria (AptCR), es la aptitud general de los sistemas cardiovascular y respiratorio que se manifiesta en la capacidad para realizar ejercicio vigoroso prolongado (10,11). La AptCR tiene diversos métodos de medición; directos, por ejemplo haciendo uso de una prueba de ergoespirometría, o indirectos como la prueba de campo de correr/caminar en doce minutos.

Por su bajo costo operacional, facilidad en su administración en ambientes específicos de práctica cotidiana y la posibilidad de evaluar un número grande de escolares, la prueba de correr/caminar de doce minutos ha sido frecuentemente utilizada para la evaluación de la AptCR ${ }^{(12)}$. Para la cual, se han establecido valores de referencia de la capacidad aeróbica para escolares de ocho a diecisiete años de edad de la región de Cariri en el noreste de Brasil (13); asimismo, se han elaborado cartas percentílicas para escolares de 6 a 17 años de edad en la región central del Perú ${ }^{(14)}$. Una baja AptCR es un marcador indirecto importante que se asocia a un mayor riesgo de enfermedades cardiovasculares ${ }^{(15,16)}$. Niveles adecuados de AptCR durante la niñez y adolescencia pueden generar beneficios a la salud durante toda la vida y condicionar la presencia de factores de riesgo cardiovascular convencionales en la vida adulta ${ }^{(17)}$.
Es por ello que en la actualidad, en algunos países, se procura establecer programas de prevención para el desarrollo de hábitos alimenticios saludables y estilos de vida activos en las escuelas, involucrando en este proceso a todos los profesionales que intervienen en la escuela (18). Ello debido a que en una encuesta de salud infantil sobre 43297 niños norteamericanos entre 10 y 17 años se encontró asociación entre la obesidad y 19 indicadores de la salud general, el funcionamiento psicosocial y trastornos específicos de salud ${ }^{(19)}$. Sin embargo, en países en vías de desarrollo, como el Perú, la falta de implementación de programas específicos para combatir el sobrepeso y la obesidad demuestran que aún no se está prestando la debida atención a este problema ${ }^{(5)}$.

Aun cuando existe información disponible a partir de estudios realizados sobre la frecuencia del exceso de peso en niños en edad escolar peruanos ${ }^{(5,20)}$, hacen falta investigaciones que, además, analicen el problema tomando en cuenta un número mayor de variables sociodemográfica y fisiológicas (como la AptCR) que pueden presentar un rol substancial para interpretar su papel relacional. El objetivo del estudio fue determinar la frecuencia del sobrepeso y obesidad, en función a los niveles de AptCR en escolares de cuatro distritos de la región central del Perú, así como analizar la asociación entre estas variables.

\section{MATERIALES Y MÉTODOS}

Se realizó un estudio trasversal, el cual forma parte del Proyecto Crecer con Salud y Esperanza, que investiga el crecimiento, desarrollo y la salud de niños, adolescentes y sus familia ${ }^{(21)}$. La información que recogida entre marzo de 2009 y diciembre de 2010.

\section{POBLACIÓN DE ESTUDIO}

La población objetivo estuvo conformada por escolares entre 6 y 17 años de edad residentes en cuatro distritos situados en regiones geográficamente diferenciadas de la región central del Perú. La edad cronológica de cada escolar se estableció de la diferencia entre la fecha de evaluación y la fecha de nacimiento obtenida de los registros de la escuela. Se incluyeron a todos los estudiantes que cumplieran el criterio de edad propuesto, se excluyó a aquellos que tuvieran alguno de los siguientes diagnósticos clínicos: discapacidad física, sensorial e intelectual señalado en el informe médico de cada participante.

\section{MUESTRA}

En primer lugar se seleccionaron cuatro distritos de la región central del Perú. En la costa se seleccionó el distrito de Barranco (de la ciudad de Lima); en la sierra, 
la ciudad de Junín (de la provincia y departamento del mismo nombre), y en la selva, los distritos de La Merced y San Ramón (de la provincia de Chanchamayo del departamento de Junín). Barranco es uno de los 43 distritos de la provincia de Lima, capital del Perú, localizado a orillas del océano Pacífico. El distrito de Junín es capital de la provincia de Junín, localizado sobre una meseta a 4107 metros de altitud en la orilla sur del lago Junín o Chinchaycocha. Los distritos de La Merced y San Ramón, que presentan una continuidad geográfica, forman parte de la provincia de Chanchamayo, localizada en el área de selva alta (500 y 2000 metros de altitud).

Se seleccionaron dieciocho instituciones educativas públicas localizadas en zona urbana, diez correspondían al nivel primario y ocho al secundario pertenecientes a los cuatro distritos previamente descritos. Se estratificó a la población estudiantil por edad, sexo e institución educativa, para así seleccionar aleatoriamente 7841 escolares (4151 mujeres y 3690 varones), como se describe en la Tabla 1. Para el cálculo del tamaño de la muestra se empleó el software WINPEPI Ver. 11.26, se consideró una frecuencia esperada de sobrepeso y obesidad del $25 \%$, con un nivel de confianza del $95 \%$ y un error del $1 \%$.

\section{MEDIDAS ANTROPOMÉTRICAS}

La altura fue medida con un estadiómetro portátil (Sanny, Model ES-2060) y los resultados tuvieron una aproximación de $0,1 \mathrm{~cm}$; el peso fue medido con una balanza digital (Pesacon, Model IP68), con una precisión de $0,1 \mathrm{~kg}$. Las mediciones fueron realizadas según técnicas estandarizadas ${ }^{(22)}$. El índice de masa corporal (IMC) fue determinado por la división del peso por el cuadrado de la altura $\left(\mathrm{kg} / \mathrm{m}^{2}\right)$ los puntos de corte utilizados para la definición del sobrepeso y obesidad fueron los propuestos por Cole et al. ${ }^{(23)}$, quienes señalan como puntos de corte el P85 y P97, respectivamente, según edad y sexo.

\section{MEDICIÓN DE LAAPTITUD CARDIORRESPIRATORIA}

La AptCR fue evaluada mediante la prueba de correr/caminar de doce minutos de la batería de la American Alliance for Health, Recreation and Dance (AAPHERD) ${ }^{(24)}$. Antes de la evaluación, se explicó a los escolares con detalle sobre las características de la prueba; y se procuró motivarlos lo suficiente para lograr su mejor desempeño. A partir de la sugerencias de Jago et al. ${ }^{(25)}$ se establecieron los puntos de corte $\left(P_{33}\right.$ y $\mathrm{P}_{66}$ ) para cada intervalo etario y género de la muestra. De acuerdo con sus resultados, los escolares fueron clasificados en la categoría de baja aptitud si estaban por debajo del Percentil $33\left(\mathrm{P}_{33}\right)$, moderada aptitud si se ubicaban entre el $P_{33}$ y $P_{66}$, y en la categoría de elevada AptCR si fue superior al $P_{66}$.

\section{CONTROL DE CALIDAD DE LA INFORMACIÓN}

El control de los datos se realizó en tres fases. En la primera, todos los miembros del equipo fueron capacitados por experimentados investigadores en el uso correcto de los procedimientos técnicos de medición corporal y administración de pruebas de desempeño motor. La segunda correspondió al estudio piloto realizado con escolares del Colegio de Aplicación en las instalaciones de la Universidad Nacional de Educación Enrique Guzmán y Valle (UNE) en marzo de 2009. Para la tercera fase se utilizó el procedimiento reliabilily in field ${ }^{(26)}$, para lo cual se aplicó un retest a escolares elegidos aleatoriamente en días alternos de evaluación. El error técnico de medida (ETM) y los coeficientes de

Tabla 1. Número de escolares de acuerdo a área geográfica, edad y sexo

\begin{tabular}{|c|c|c|c|c|c|c|c|c|}
\hline \multirow[b]{2}{*}{$\begin{array}{c}\text { Edad } \\
\text { (años) }\end{array}$} & \multicolumn{2}{|c|}{ Costa } & \multicolumn{2}{|c|}{ Sierra } & \multicolumn{2}{|c|}{$\begin{array}{c}\text { Selva } \\
\end{array}$} & \multicolumn{2}{|c|}{ Total } \\
\hline & $\begin{array}{c}\text { Mujer } \\
\text { N. }{ }^{\circ}(\%)\end{array}$ & $\begin{array}{c}\text { Varón } \\
\mathbf{N} .^{\circ}(\%)\end{array}$ & $\begin{array}{c}\text { Mujer } \\
\text { N. }{ }^{\circ}(\%)\end{array}$ & $\begin{array}{c}\text { Varón } \\
\text { N. }{ }^{\circ}(\%)\end{array}$ & $\begin{array}{c}\text { Mujer } \\
\text { N. }{ }^{\circ}(\%)\end{array}$ & $\begin{array}{c}\text { Varón } \\
\text { N. }{ }^{\circ}(\%)\end{array}$ & $\begin{array}{c}\text { Mujer } \\
\text { N. }{ }^{\circ}(\%)\end{array}$ & $\begin{array}{c}\text { Varón } \\
\mathrm{N}^{\circ}{ }^{\circ}(\%)\end{array}$ \\
\hline 6 & $109(4,9)$ & $80(3,6)$ & $38(2,1)$ & $48(2,7)$ & $162(4,2)$ & $153(3,9)$ & $309(3,9)$ & $281(3,6)$ \\
\hline 7 & $76(3,5)$ & $52(2,4)$ & $55(3,1)$ & $47(2,6)$ & $140(3,6)$ & $192(4,9)$ & $271(3,5)$ & $291(3,7)$ \\
\hline 8 & $82(3,7)$ & $64(2,9)$ & $47(2,6)$ & $60(3,4)$ & $178(4,6)$ & $174(4,5)$ & $307(3,9)$ & $298(3,8)$ \\
\hline 9 & $119(5,4)$ & $76(3,5)$ & $65(3,6)$ & $76(4,2)$ & $187(4,9)$ & $184(4,8)$ & $371(4,7)$ & $336(4,3)$ \\
\hline 10 & $85(3,9)$ & $84(3,8)$ & $70(3,9)$ & $71(3,9)$ & $218(5,7)$ & $189(4,9)$ & $373(4,8)$ & $344(4,4)$ \\
\hline 11 & $111(5,1)$ & $114(5,2)$ & $73(4,1)$ & $55(3,1)$ & $193(5,0)$ & $189(4,9)$ & $377(4,8)$ & $358(4,6)$ \\
\hline 12 & $92(4,2)$ & $119(5,4)$ & $134(7,5)$ & $90(5,0)$ & $237(6,2)$ & $144(3,7)$ & $463(5,9)$ & $353(4,5)$ \\
\hline 13 & $69(3,1)$ & $64(2,9)$ & $76(4,2)$ & $92(5,1)$ & $212(5,5)$ & $144(3,7)$ & $357(4,6)$ & $300(3,8)$ \\
\hline 14 & $125(5,7)$ & $102(4,6)$ & $109(6,1)$ & $102(5,7)$ & $187(4,9)$ & $120(3,1)$ & $421(5,4)$ & $324(4,1)$ \\
\hline 15 & $110(5,0)$ & $142(6,5)$ & $98(5,5)$ & $85(4,7)$ & $150(3,9)$ & $151(3,9)$ & $358(4,6)$ & $378(4,8)$ \\
\hline 16 & $138(6,3)$ & $82(3,7)$ & $105(5,9)$ & $84(4,7)$ & $132(3,4)$ & $118(3,1)$ & $375(4,8)$ & $284(3,6)$ \\
\hline 17 & $64(2,9)$ & $41(1,9)$ & $60(3,4)$ & $52(2,9)$ & $45(1,2)$ & $50(1,3)$ & $169(2,2)$ & $143(1,8)$ \\
\hline Total & $1180(53,6)$ & $1020(46,4)$ & $930(51,9)$ & $862(48,1)$ & $2041(53,0)$ & $1808(46,9)$ & $4151(52,9)$ & $3690(47,1)$ \\
\hline
\end{tabular}


correlación intraclase $(R)$ basados en ANOVA fueron utilizados para estimar el grado de precisión y la proporción de la variación en las mediciones. Para la altura se encontró un $E T M=0,2 \mathrm{~cm}$ y un $R=0,96$; para el peso un $E T M=0,1 \mathrm{~kg}$ y un $R$ de 0,98 , y para la prueba de correr/caminar 12 minutos un $\mathrm{R}=0,87$.

\section{ANÁLISIS ESTADÍSTICO}

Las características de la muestra de estudio se presentan como frecuencias, porcentajes o como medias y desviación estándar, según corresponda. Se utilizó el análisis de variancia de un solo factor (ANOVA) para determinar las diferencias de la AptCR entre los grupos de estatus ponderal en cada intervalo etario, género, nivel educativo y área geográfica, seguido de un análisis de Tukey, según fuera necesario. La regresión logística fue utilizada para examinar las asociaciones de las categorías ponderales con la edad, el sexo, nivel educativo, área geográfica y las categorías de AptCR. Todos los análisis estadísticos se realizaron con el programa estadístico SPSS ver 20.0 (SPSS, Inc., Chicago, IL). El nivel de significancia fue fijado en $5 \%$.

\section{ASPECTOS ÉTICOS}

El Proyecto Crecer con Salud y Esperanza fue sometido a valoración y ha obtenido la aprobación del Instituto de Investigación de la Universidad Nacional de Educación Enrique Guzmán y Valle. Después de contactos iniciales con autoridades educativas, de salud y políticas de cada ciudad, se solicitó un permiso oficial de quienes dirigen las instituciones participantes en el estudio. Se obtuvo las autorizaciones de los padres y el consentimiento de cada escolar. La identidad de los participantes se mantuvo en reserva en la fase de análisis de datos.

\section{RESULTADOS}

La frecuencia de escolares normoponderales, con sobrepeso y obesidad está descrita en la Tabla 2 . Se encontró que la frecuencia de sobrepeso/obesidad en ambos sexos es similar $(20,9$ y $20,1 \%$ en mujeres y varones, respectivamente). Los escolares que residen en la sierra presentan frecuencias disminuidas de sobrepeso/obesidad (6,9 y $3,6 \%$ en mujeres y varones, respectivamente). Por otro lado, el $27,8 \%$ de mujeres y $29,9 \%$ de varones que demuestran una AptCR baja pertenecen a la categoría ponderal de sobrepeso / obesidad.

En la Tabla 3, se resume los resultados de la AptCR de acuerdo con el sexo, la edad, el nivel educativo y área geográfica en cada categoría ponderal. Los datos demuestran diferencias significativas $(p<0,01)$ cuando se
Tabla 2. Sobrepeso y obesidad en escolares de cuatro distritos de la región central del Perú

\begin{tabular}{|c|c|c|c|}
\hline & Normoponderal & Sobrepeso & Obesidad \\
\hline & N. ${ }^{\circ}(\%)$ & N. ${ }^{\circ}(\%)$ & N. ${ }^{\circ}(\%)$ \\
\hline \multicolumn{4}{|l|}{ Sexo } \\
\hline Mujer & $3284(79,1)$ & $714(17,2)$ & $153(3,7)$ \\
\hline Varón & $2951(80,0)$ & $582(15,8)$ & $157(4,3)$ \\
\hline \multicolumn{4}{|l|}{ Edad } \\
\hline 6 & $443(75,1)$ & $108(18,3)$ & $39(6,6)$ \\
\hline 7 & $423(75,3)$ & $103(18,3)$ & $36(6,4)$ \\
\hline 8 & $471(77,9)$ & $102(16,9)$ & $32(5,3)$ \\
\hline 9 & $523(74,0)$ & $141(19,9)$ & $43(6,1)$ \\
\hline 10 & $572(79,8)$ & $113(15,8)$ & $32(4,5)$ \\
\hline 11 & $534(72,7)$ & $162(22,0)$ & $39(5,3)$ \\
\hline 12 & $641(78,6)$ & $145(17,8)$ & $30(3,7)$ \\
\hline 13 & $541(82,3)$ & $97(14,8)$ & $19(2,9)$ \\
\hline 14 & $626(84,0)$ & $102(13,7)$ & $17(2,3)$ \\
\hline 15 & $634(86,1)$ & $92(12,5)$ & $10(1,4)$ \\
\hline 16 & $561(85,1)$ & $89(13,5)$ & $9(1,4)$ \\
\hline 17 & $266(85,3)$ & $42(13,5)$ & $4(1,3)$ \\
\hline \multicolumn{4}{|c|}{ Nivel educativo } \\
\hline \multicolumn{4}{|c|}{ Primaria } \\
\hline Mujer & $1614(76,3)$ & $386(18,2)$ & $116(5,5)$ \\
\hline Varón & $1524(76,8)$ & $355(17,9)$ & $105(5,3)$ \\
\hline \multicolumn{4}{|c|}{ Secundaria } \\
\hline Mujer & $1670(82,1)$ & $328(16,1)$ & $37(1,8)$ \\
\hline Varón & $1427(83,6)$ & $227(13,3)$ & $52(3,0)$ \\
\hline \multicolumn{4}{|c|}{ Área geográfica } \\
\hline \multicolumn{4}{|c|}{ Costa } \\
\hline Mujer & $764(64,7)$ & $312(26,4)$ & $104(8,8)$ \\
\hline Varón & $608(59,6)$ & $303(29,7)$ & $109(10,7)$ \\
\hline \multicolumn{4}{|l|}{ Sierra } \\
\hline Mujer & $866(93,1)$ & $63(6,8)$ & $1(0,1)$ \\
\hline Varón & $831(96,4)$ & $31(3,6)$ & $0(0,0)$ \\
\hline \multicolumn{4}{|l|}{ Selva } \\
\hline Mujer & $1654(81,0)$ & $339(16,6)$ & $48(2,4)$ \\
\hline Varón & $1512(83,6)$ & $248(13,7)$ & $48(2,7)$ \\
\hline \multicolumn{4}{|c|}{ Aptitud cardiorrespiratoria } \\
\hline \multicolumn{4}{|c|}{ Elevada } \\
\hline Mujer & $786(89,8)$ & $85(9,7)$ & $4(0,5)$ \\
\hline Varón & $1298(88,1)$ & $161(10,9)$ & $14(1,0)$ \\
\hline \multicolumn{4}{|l|}{ Moderada } \\
\hline Mujer & $1218(80,3)$ & $264(17,4)$ & $35(2,3)$ \\
\hline Varón & $738(81,2)$ & $139(15,3)$ & $32(3,5)$ \\
\hline \multicolumn{4}{|l|}{ Baja } \\
\hline Mujer & $1115(72,2)$ & $323(20,9)$ & $106(6,9)$ \\
\hline Varón & $619(70,1)$ & $193(21,9)$ & $71(8,0)$ \\
\hline
\end{tabular}

comparan las medias correspondientes a las categorías ponderales en ambos sexos y en cada intervalo etario. En todos los casos, los normoponderales presentaron valores superiores a las demás categorías ponderales y las medias de los escolares con sobrepeso fueron mayores en comparación con el de los obesos. Resultados similares se evidenciaron al realizar comparaciones en mujeres y varones pertenecientes a los niveles educativos primaria 
Tabla 3. Diferencias de las medias de aptitud cardiorrespiratoria en escolares de cuatro distritos de la región central del Perú

\begin{tabular}{|c|c|c|c|c|c|}
\hline & \multirow{2}{*}{ N. ${ }^{\circ}$} & \multirow{2}{*}{$\begin{array}{c}\text { Normoponderal } \\
\text { Media } \pm \text { DE }\end{array}$} & \multirow{2}{*}{$\begin{array}{c}\text { Sobrepeso } \\
\text { Media } \pm \text { DE }\end{array}$} & \multirow{2}{*}{$\begin{array}{c}\text { Obesidad } \\
\text { Media } \pm \text { DE }\end{array}$} & \multirow{2}{*}{$p$} \\
\hline & & & & & \\
\hline \multicolumn{6}{|l|}{ Sexo } \\
\hline Mujer & 4151 & $1393 \pm 358$ & $1279 \pm 317$ & $1137 \pm 281$ & $0,001^{*, t}$ \\
\hline Varón & 3690 & $1625 \pm 487$ & $1430 \pm 443$ & $1218 \pm 380$ & $0,001^{*}, t$ \\
\hline \multicolumn{6}{|l|}{ Edad } \\
\hline 6 & 590 & $1119 \pm 308$ & $1016 \pm 292$ & $969 \pm 156$ & $0,002^{*}, t$ \\
\hline 7 & 562 & $1291 \pm 324$ & $1197 \pm 305$ & $1032 \pm 135$ & $0,001^{*}, t$ \\
\hline 8 & 605 & $1357 \pm 329$ & $1273 \pm 256$ & $1089 \pm 203$ & $0,001^{*}, t$ \\
\hline 9 & 707 & $1395 \pm 322$ & $1303 \pm 309$ & $1162 \pm 291$ & $0,001^{*}, t$ \\
\hline 10 & 717 & $1545 \pm 434$ & $1447 \pm 508$ & $1318 \pm 403$ & $0,005^{\dagger}$ \\
\hline 11 & 735 & $1555 \pm 462$ & $1388 \pm 427$ & $1287 \pm 476$ & $0,001^{*}, t$ \\
\hline 12 & 816 & $1518 \pm 404$ & $1368 \pm 310$ & $1256 \pm 290$ & $0,001^{*}, t$ \\
\hline 13 & 657 & $1499 \pm 412$ & $1386 \pm 384$ & $1169 \pm 280$ & $0,001^{*}, t$ \\
\hline 14 & 745 & $1579 \pm 434$ & $1372 \pm 354$ & $1272 \pm 399$ & $0,001^{*}, t$ \\
\hline 15 & 736 & $1645 \pm 459$ & $1483 \pm 398$ & $1442 \pm 286$ & $0,007^{*}$ \\
\hline 16 & 659 & $1644 \pm 478$ & $1441 \pm 356$ & $1300 \pm 348$ & $0,001^{*}$ \\
\hline 17 & 312 & $1711 \pm 505$ & $1549 \pm 421$ & $1197 \pm 323$ & $0,037^{*}, \dagger$ \\
\hline \multicolumn{6}{|c|}{ Nivel educativo } \\
\hline \multicolumn{6}{|c|}{ Primaria } \\
\hline Mujer & 2116 & $1361 \pm 361$ & $1247 \pm 320$ & $1133 \pm 295$ & $0,001^{*}, t$ \\
\hline Varón & 1984 & $1500 \pm 476$ & $1349 \pm 457$ & $1164 \pm 376$ & $0,001^{*}, t$ \\
\hline \multicolumn{6}{|c|}{ Secundaria } \\
\hline Mujer & 2035 & $1422 \pm 352$ & $1315 \pm 310$ & $1149 \pm 238$ & $0,001^{*}, t$ \\
\hline Varón & 1706 & $1757 \pm 463$ & $1560 \pm 387$ & $1345 \pm 365$ & $0,001^{*}, t$ \\
\hline \multicolumn{6}{|c|}{ Área geográfica } \\
\hline \multicolumn{6}{|c|}{ Costa } \\
\hline Mujer & 1180 & $1320 \pm 252$ & $1250 \pm 218$ & $1110 \pm 179$ & $0,001^{*}, t$ \\
\hline Varón & 1020 & $1407 \pm 434$ & $1305 \pm 387$ & $1129 \pm 263$ & $0,001^{*}, t$ \\
\hline \multicolumn{6}{|l|}{ Sierra } \\
\hline Mujer & 930 & $1579 \pm 290$ & $1577 \pm 278$ & $1315 \pm 318$ & 0,436 \\
\hline Varón & 862 & $1794 \pm 486$ & $1613 \pm 381$ & - & $0,043^{*}$ \\
\hline \multicolumn{6}{|l|}{ Selva } \\
\hline Mujer & 2041 & $1327 \pm 395$ & $1249 \pm 368$ & $1188 \pm 418$ & $0,001^{*}, t$ \\
\hline Varón & 1808 & $1596 \pm 471$ & $1524 \pm 469$ & $1360 \pm 486$ & $0,001^{\dagger}$ \\
\hline
\end{tabular}

Nota: todas las medias están expresadas en metros y corresponden a la prueba caminar/correr en 12 minutos.

DE: desviación estándar.

* Tukey post hoc: $p<0,05$ normoponderal comparado con sobrepeso

† Tukey post hoc: $p<0,05$ normoponderal comparado con obesidad

y secundaria; asimismo, en las tres áreas geográficas analizadas, con la sola excepción del sexo femenino que corresponde a la sierra $(p=0,44)$. Los resultados revelan que los escolares que pertenecen a la categoría de sobrepeso y obesidad presentan valores más bajos de AptCR en relación con los normoponderales.

Las asociaciones entre las frecuencias de sobrepeso y obesidad con la edad, el sexo, nivel educativo, área geográfica y la AptCR son mostrados en la Tabla 4. Se encontró que con el aumento de la edad existe menor riesgo de presentar sobrepeso y obesidad [OR=0,95 (IC95\%: 0,91-0,98) y OR= 0,88 (IC95\%: 0,83-0,93), respectivamente]. Se encontró que las mujeres en comparación con los varones tienen menor probabilidad de ser obesas [OR= 0,80 (IC95\%: 0,65-0,99)]. Estudiar en uno de los tres niveles educativos parece no influir en el estatus ponderal de forma significativa. Por otro lado, el residir en un área geográfica determinada influyó en el estatus ponderal $(p<0,01)$. Los escolares que viven en la costa (Barranco) tienen aproximadamente tres veces más probabilidad de presentar sobrepeso [OR= 2,77 (IC95\%: 2,40-3,20)] y son cinco veces más propensos a ser obesos [OR=4,67 (IC95\%: 3,55-6,14]; mientras que los que habitan en la sierra (Junín) demuestran pequeñas probabilidades de tener sobrepeso [OR=0,34 (IC95\%: 0,27-0,41)] u obesidad [OR=0,03 (IC95\%: 0,01-0,20)].

La AptCR es una variable que expresa una asociación directa con las categorías del estatus ponderal, se 
Tabla 4. Análisis bivariado y multivariado para sobrepeso y obesidad en escolares de cuatro distritos de la región central del Perú

\begin{tabular}{|c|c|c|c|c|c|c|}
\hline \multirow{4}{*}{ Variables explicativas } & \multicolumn{6}{|c|}{ Categorías ponderales } \\
\hline & \multicolumn{3}{|c|}{ Sobrepeso } & \multicolumn{3}{|c|}{ Obesidad } \\
\hline & OR & ORa & & OR & ORa & \\
\hline & (IC95\%) & (IC95\%) & $\mathrm{p}$ & (IC95\%) & (IC95\%) & $\mathbf{p}$ \\
\hline Edad & $\begin{array}{c}0,95 \\
(0,94-0,96)\end{array}$ & $\begin{array}{c}0,95 \\
(0,91-0,98)\end{array}$ & $<0,01$ & $\begin{array}{c}0,85 \\
(0,82-0,88)\end{array}$ & $\begin{array}{c}0,88 \\
(0,83-0,93)\end{array}$ & $<0,01$ \\
\hline \multicolumn{7}{|l|}{ Sexo } \\
\hline Mujer & $\begin{array}{c}1,10 \\
(0,99-1,23)\end{array}$ & $\begin{array}{c}0,99 \\
(0,87-1,14)\end{array}$ & 0,92 & $\begin{array}{c}0,88 \\
(0,70-1,10)\end{array}$ & $\begin{array}{c}0,80 \\
(0,65-0,99)\end{array}$ & 0,09 \\
\hline \multicolumn{7}{|l|}{ Nivel educativo* } \\
\hline Primaria & $\begin{array}{c}1,32 \\
(1,17-1,49)\end{array}$ & $\begin{array}{c}0,94 \\
(0,74-1,18)\end{array}$ & 0,57 & $\begin{array}{c}2,45 \\
(1,91-3,15)\end{array}$ & $\begin{array}{c}1,05 \\
(0,65-1,70)\end{array}$ & 0,83 \\
\hline \multicolumn{7}{|l|}{ Área geográfica** } \\
\hline Sierra & $\begin{array}{c}0,30 \\
(0,24-0,37)\end{array}$ & $\begin{array}{c}0,34 \\
(0,27-0,41)\end{array}$ & $<0,01$ & $\begin{array}{c}0,02 \\
(0,01-0,14)\end{array}$ & $\begin{array}{c}0,03 \\
(0,01-0,20)\end{array}$ & $<0,01$ \\
\hline Costa & $\begin{array}{c}2,42 \\
(2,12-2,75)\end{array}$ & $\begin{array}{c}2,77 \\
(2,40-3,20)\end{array}$ & $<0,01$ & $\begin{array}{c}5,12 \\
(4,01-6,57)\end{array}$ & $\begin{array}{c}4,67 \\
(3,55-6,14)\end{array}$ & $<0,01$ \\
\hline \multicolumn{7}{|l|}{ Aptitud cardiorrespiratoria $^{\dagger}$} \\
\hline Moderada & $\begin{array}{c}1,75 \\
(1,47-2,07)\end{array}$ & $\begin{array}{c}1,37 \\
(1,15-1,65)\end{array}$ & $<0,01$ & $\begin{array}{c}3,97 \\
(2,35-6,70)\end{array}$ & $\begin{array}{c}2,65 \\
(1,55-4,55)\end{array}$ & $<0,01$ \\
\hline Baja & $\begin{array}{c}2,52 \\
(2,14-2,97) \\
\end{array}$ & $\begin{array}{c}1,59 \\
(1,32-1,90)\end{array}$ & $<0,01$ & $\begin{array}{c}11,82 \\
(7,25-19,27)\end{array}$ & $\begin{array}{c}5,01 \\
(3,01-8,35) \\
\end{array}$ & $<0,01$ \\
\hline
\end{tabular}

ORa: odds ratio ajustado

Categorías de referencia: * secundaria, ${ }^{* *}$ selva, ${ }^{\dagger}$ elevada

encontró que los niños y adolescentes con baja AptCR se asociaron significativamente a obesidad $[\mathrm{OR}=11,82$ (IC95\%: 7,25-19,27)]; mientras aquellos que presentan una moderada AptCR son cuatro veces más propensos a ser obesos [OR= 3,97 (IC95\%: 2,35 - 6,70)]. Sin embargo, el modelo de regresión múltiple mostro que los escolares con un bajo nivel de AptCR tienen una vez y media la probabilidad de tener sobre peso y quintuplican su probabilidad de ser obsesos (Tabla 4).

\section{DISCUSIÓN}

Los resultados del análisis de la frecuencia del sobrepeso y obesidad en relación con el sexo, la edad, el nivel educativo y área geográfica señalan que, aproximadamente uno de cada cinco niños en edad escolar tienen sobrepeso u obesidad, verificándose frecuencias similares en ambos sexos $(20,9 \%$ en niñas y $20,1 \%$ en niños); resultado similar al encontrado en escolares entre 6 y 11 años de edad en distritos del Cono Este de Lima Metropolitana ${ }^{(20)}$. Es de resaltar que en el intervalo etario de 6 a los 9 años las frecuencias de sobrepeso y obesidad encontradas en nuestro estudio $(25,3 \%)$ son ligeramente mayores a las halladas en la ENAHO (24,4\%); mientras que en el intervalo de 10 a 19 años, las diferencias son mayores $(18,5 \% \text { frente a } 14,2 \%)^{(5)}$.

Por otro lado, los escolares de la ciudad de Junín presentan una baja frecuencia de sobrepeso y obesidad $(5,3 \%)$, seguido por los que residen en Chanchamayo y Barranco $(17,7$ y $37,8 \%$, respectivamente). Similar tendencia se encontró en la ENAHO, donde en el intervalo etario de cinco a nueve años se halló un $36,2 \%$ de sobrepeso y obesidad en Lima Metropolitana y entre los 10 y 19 años un 18,1\%. Para la sierra, en los mismos intervalos etarios, reportaron un 14,8 y $9,6 \%$ de niños y adolescentes con sobrepeso y obesidad, y en la selva fue de 14,2 y $9,3 \%$. Por otro lado, Yépez et al. (4) encontraron en adolescentes ecuatorianos que residen en la costa y sierra un 24,7 y $17,7 \%$ de sobrepeso y obesidad, respectivamente.

Estas diferencias son atribuidas, en parte, al mayor nivel de urbanización y menor pobreza extrema que se observa en los distritos que se ubican en la costa, que da lugar a modificaciones de los estilos de vida relativos a los patrones de alimentación y de actividad física ${ }^{(5)}$. Las comparaciones con otros estudios deben realizarse con reserva, puesto que, diferencias metodológicas relativas al empleo de diferentes puntos de corte, grandes diferencias entre las poblaciones de referencia y el periodo de recolección de datos pueden inducir a confundir los resultados ${ }^{(27)}$.

La obesidad tiene un componente genético relevante ${ }^{(28)}$; del mismo modo, los factores ambientales demuestran una elevada importancia que intervienen de forma compleja y desigual entre o dentro de los países ${ }^{(6)}$. El incremento de la frecuencia del sobrepeso/obesidad en niños y adolescentes peruanos podría atribuirse a cambios diferenciados en los estilos de vida en diversas 
áreas geográficas: Barranco presenta una densidad poblacional de 10181,08 hab./km², inseguridad pública creciente, y una falta de infraestructura deportivorecreacional pública accesible a la población escolar, situación que estaría contribuyendo en la adopción de un estilo de vida sedentario; en tanto, Junín tiene una densidad poblacional de 12,79 hab. $/ \mathrm{km}^{2}$ y Chanchamayo de 27,8 hab. $/ \mathrm{km}^{2}$ situación que predispone a que la mayoría de su población escolar demuestre patrones y niveles de actividad física (recreativas, tareas domésticas y de apoyo en actividades agrícolas en el campo) con intensidad, frecuencia y duración superior a sus pares costeños. Asimismo, los escolares de Barranco son más propensos a adquirir hábitos alimentarios nocivos, por ejemplo, consumir alimentos altamente calóricos y pobremente nutricionales; mientras que, en Junín y Chanchamayo, las familias aún mantendrían la prioridad por el consumo de productos oriundos.

Los resultados del presente estudio revelaron una baja frecuencia de sobrepeso y obesidad en escolares de ambos sexos que manifestaron niveles elevados de AptCR (11,05\%), en comparación con sus pares con bajos niveles de AptCR (28,9\%). Estos resultados concuerdan con lo observado en el desempeño de la prueba de correr/caminar en doce minutos (Tabla 3), que expresa el desarrollo de la AptCR y viene a ser uno de los componentes más importantes de la aptitud física asociada a la salud. Se verificó que los normoponderales en cuanto al sexo, la edad, el nivel educativo y área geográfica presentan en casi la totalidad de las comparaciones una mayor capacidad aeróbica en relación con los escolares con sobrepeso y obesidad.

La adopción de un estilo de vida sedentario y patrones nutricionales inadecuados conlleva a una reducción drástica de actividad física moderada a vigorosa en escolares, asociándose así el sobrepeso y la obesidad a bajos niveles de actividad física que se refleja en una disminución de los niveles de aptitud física en todos sus componentes, especialmente de la AptCR $(6,16,17)$. En una muestra de adolescentes del presente estudio se verificó, que una proporción apropiada del peso corporal para la estatura (índice ponderal recíproco), es un predictor adecuado para el desarrollo de la AptCR. Es decir, los escolares con elevada AptCR en relación con sus pares con baja AptCR muestran frecuencias reducidas de sobrepeso/obesidad que les permite ejecutar tareas motoras con intensidad, frecuencia y duración superior que asegura el mantenimiento o la mejora de su capacidad aeróbica.

Resultados del Quebec Family Study demostraron que adolescentes de 9 a 18 años de edad, con valores altos de IMC y baja aptitud aeróbica presentaban un perfil de riesgo de enfermedades cardiovasculares más pobre en comparación con aquellos con IMC bajo y alta aptitud que tenían un mejor perfil ${ }^{29)}$. Por otro lado, cuando se examinó la influencia de la aptitud aeróbica y el IMC en los valores del síndrome metabólico (SM) en niños de 7 a 9 años, reportaron que niveles altos de AptCR modificaron el impacto que tuvo el IMC en la puntuación del SM (30). Estos estudios sugieren que promover el desarrollo de la AptCR durante la infancia es importante para reducir el riesgo de comorbilidades relacionadas con la obesidad.

En cuanto a la asociación entre el estatus ponderal y el área geográfica, los resultados revelan que residir en la costa (Barranco) conlleva a tener mayor probabilidad de presentar sobrepeso u obesidad en comparación con los escolares que habitan en la sierra (Junín) y selva (Chanchamayo). Diversos estudios' han demostrado esta asociación. Por ejemplo, los resultados encontrados en la ENAHO ${ }^{(5)}$ indican que los habitantes de Lima Metropolitana fueron más prevalentes en relación con los de la sierra y selva.

Bajos niveles de AptCR durante la niñez y adolescencia es un indicador que refleja una asociación significativa con mayores probabilidades para que los escolares puedan presentar sobrepeso u obesidad ${ }^{(1,18)}$. Los resultados encontrados en el presente estudio demuestran el efecto protector de la AptCR para con las enfermedades no transmisibles que son la causa más común de morbilidad y mortalidad en adultos similar a lo reportado por otros estudios.

Estos resultados conducen a reflexionar sobre la necesidad de que las autoridades políticas, de salud y educativas implementen programas de intervención permanentes en las escuelas que involucre a todos los profesionales que forman parte de ella, así como a los padres, dado que la prevención debe empezar desde el hogar. La adopción de hábitos alimentarios saludables, el uso adecuado de los tiempos libres en el hogar y la escuela, así como sesiones de educación física que promueva la adquisición y consolidación progresiva de destrezas motoras y el incremento y mantenimiento de los niveles de aptitud física, dando prioridad a ejercicios físicos de moderada a elevada intensidad que producen mayores cambios en la AptCR.

Algunas limitaciones deben ser reconocidas; la primera está referida a su naturaleza transversal, con una muestra que proviene de cuatro distritos ubicados en tres áreas geográficas diferenciadas en la región central del Perú, que condiciona de la interpretación y generalización de sus resultados. La segunda, es la falta de información sobre los patrones alimentarios y de actividad física que no permite llegar a conclusiones 
firmes sobre la influencia ambiental subyacente. Sin embargo, esta información es relevante porque ha permitido identificar y analizar los efectos de algunos factores asociados con el sobrepeso y obesidad.

En conclusión, los escolares de la región central del Perú presentan frecuencias de sobrepeso y obesidad que pueden tener repercusiones negativas en el estado de salud futura. Niveles bajos de aptitud cardiorrespiratoria están asociados significativamente al sobrepeso y a la obesidad, al igual que los escolares que residen en distritos costeros.

Agradecimientos: a la Fundación para la Ciencia y Tecnología de Portugal por la beca de manutención; a los escolares que participaron en este estudio; a los estudiantes y profesores de educación física de la UNE, que contribuyeron en la recolección de datos; y al Dr. Gastón Beunen, quien fue uno de los promotores del Proyecto Crecer con Salud y Esperanza.

Contribuciones de autoría: ABV y JARM participaron en la concepción y el diseño del artículo. ABV realizó el análisis y la interpretación de los resultados y la redacción del artículo. JARM efectuó la asesoría estadística, la revisión crítica del artículo. Ambos autores aprobaron la versión final del artículo.

Fuentes de financiamiento: Fundación para la Ciencia y Tecnología de Portugal.

Conflictos de interés: los autores declaran no tener conflictos de interés.

\section{REFERENCIAS BIBLIOGRÁFICAS}

1. Organización Mundial de la Salud. Temas de salud [Internet]. Ginebra: Organización Mundial de la Salud; 2013 [citado el 19 de febrero de 2013]. Disponible en: http://www.who.int/mediacentre/ factsheets/fs311/es/index.html

2. Chen LJ, Fox KR, Haase A, Wang JM. Obesity, fitness and health in Taiwanese children and adolescents. Eur J Clin Nutr. 2006;60(12):1367-75.

3. International Association for the Study of Obesity. World map of obesity [Internet]. London: International Association for the Study of Obesity; 2013 [citado el 20 de febrero de 2013]. Disponible en: http://www.iaso.org/resources/worldmap-obesity/?map=children

4. Yepez R, Carrasco F, Baldeón ME. Prevalencia de sobrepeso y obesidad en estudiantes adolescentes ecuatorianos del área urbana. ALAN. 2008;58(2):139-43.

5. Alvarez-Dongo D, Sanchez-Abanto J, Gomez-Guizado G, Tarqui-Mamani C. Sobrepeso y obesidad: prevalencia y determinantes sociales del exceso de peso en la población peruana (20092010). Rev Peru Med Exp Salud Publica. 2012;29(3):303-13.

6. Brophy S, Rees A, Knox G, Baker J, Thomas NE. Child fitness and father's BMI are important factors in childhood obesity: a school based cross-sectional study. PloS One. 2012;7(5):e36597. doi: 10.1371/journal.pone.0036597.

7. Martins D, Maia J, Seabra A, Garganta R, Lopes V, Katzmarzyk P, et al. Correlates of changes in BMI of children from the Azores islands. Int J Obesity. 2010;34(10):1487-93.
8. Pereira S, Seabra A, Silva R, Zhu W, Beunen G, Maia J. Correlates of health-related physical fitness levels of Portuguese children. Int J Pediatr Obes. 2011;6(53-59).

9. Bouchard C, Shepard R. Physical Activity, Fitness, and Health: The Model and Key Concepts. In: Bouchard C, Shepard R, Stephens T, editors. Physical Activity, Fitness and Health. Champaign: Human Kinetics; 1993. p. 11-23.

10. Ortega FB, Ruiz JR, Castillo MJ, Sjöström M. Physical fitness in childhood and adolescence: a powerful marker of health. Int J Obes (Lond). 2008;32(1):1-11.

11. Malina R, Bouchard C, Bar-Or O. Growth, maturation, and physical activity. 2nd ed. Champaign: Human Kinetics; 2004.

12. Safrit MJ. Complete guide to youth fitness testing. Champaign: Human Kinetics; 1995.

13. Silva S, Beunen G, Maia J. Valores normativos do desempenho motor de crianças e adolescentes: o estudo longitudinalmisto do Cariri. Rev bras educ fís esporte. 2011;25(1):111-25.

14. Bustamante A, Beunen G, Maia J. Valoración de la aptitud física en niños y adolescentes: construcción de cartas percentílicas para la región central del Perú. Rev Peru Med Exp Salud Publica. 2012;29(2):188-97.

15. Ruiz JR, Ortega FB, Gutierrez A, Meusel D, Sjöström M, Castillo MJ. Healthrelated fitness assessment in childhood and adolescence: a European approach based on the AVENA, EYHS and HELENA studies. Journal of Public Health. 2006;14(5):269-77.
16. Lee CD, Blair SN, Jackson AS. Cardiorespiratory fitness, body composition, and all-cause and cardiovascular disease mortality in men. Am J Clin Nutr. 1999;69(3):373-80.

17. Twisk JW, Kemper HC, van Mechelen W. The relationship between physical fitness and physical activity during adolescence and cardiovascular disease risk factors at adult age. The Amsterdam Growth and Health Longitudinal Study. Int J Sports Med 2002;23(S1):8-14.

18. Halfon N, Larson K, Slusser W. Associations between obesity and comorbid mental health, developmental, and physical health conditions in a nationally representative sample of US children aged 10 to 17. Acad Pediatr. 2013;13(1):6-13.

19. Yager Z, O'Dea JA. The role of teachers and other educators in the prevention of eating disorders and child obesity: what are the issues? Eat Disord. 2005;13(3):261-78.

20. Bustamante A, Seabra AF, Garganta RM, Maia JA. Efectos de la actividad física y del nivel socioeconómico en el sobrepeso y obesidad de escolares, Lima Este 2005. Rev Peru Med Exp Salud Publica. 2007;24(2):121-8.

21. Bustamante A, Beunen G, Maia J. ¿Como crecen y se desarrollan los niños y adolescentes en La Merced y San Ramón? En: Alcances para la Educación Física, el Deporte y la Salud. Lima: Universidad Nacional de Educación; 2011. p. 174.

22. Lohman $\mathrm{T}$, Roche A, Martorell $\mathrm{R}$. Anthropometric Standardization Reference Manual. Champaign, IL: Human Kinetics Books; 1988. 
23. Cole TJ, Bellizzi MC, Flegal KM, Dietz WH. Establishing a standard definition for child overweight and obesity worldwide: international survey. BMJ. 2000;320(7244):1240-3.

24. American Alliance for Health PE, Recreation and Dance (AAHPERD). Health related physical fitness manual. Washington: AAHPERD; 1980.

25. Jago R, Drews KL, McMurray RG, Baranowski T, Galassetti P, Foster GD, et al. BMI change, fitness change and cardiometabolic risk factors among 8th grade youth. Pediatr Exerc Sci. 2013;25(1):52-68.

26. Streiner DL, Norman GR. Health measurement scales. A practical guide to ther development and use. 4th ed. Oxford.: Oxford Unversity Press; 2008.
27. Valerio G, Scalfi L, De Martino C, Franzese A, Tenore A, Contaldo F. Comparison between different methods to assess the prevalence of obesity in a sample of Italian children. J Pediatr Endocr Met. 2003;16(2):211-6.

28. Bouchard C. Discussion: Heredity, fitness and health. In: Bouchard C, Shepard RJ, Stephens T, Sutton JR, McPherson $\mathrm{BD}$, editors. Exercise, fitness and health. Champaign,: Human Kinetics; 1990. p. 147-53.

29. Eisenmann JC, Katzmarzyk PT, Perusse L, Tremblay A, Després JP, Bouchard C. Aerobic fitness, body mass index, and CVD risk factors among adolescents: the Québec family study. Int J Obes (Lond). 2005;29(9):1077-83.
30. DuBose KD, Eisenmann JC, Donnelly JE. Aerobic fitness attenuates the metabolic syndrome score in normal-weight, at-riskfor-overweight, and overweight children. Pediatrics. 2007;120(5):e1262-8.

Correspondencia: Alcibiades Bustamante Valdivia

Dirección: Universidade do Porto, Faculdade de Desporto, Laboratório de Cineantropometria e Estatística Aplicada, Rua Plácido Costa 91,4200.450, Porto, Portugal. Teléfono: (351) 938405522

Correo electrónico:buanta2609@yahoo.es

\section{PRÓXIMAMENTE DISPONIBLE}

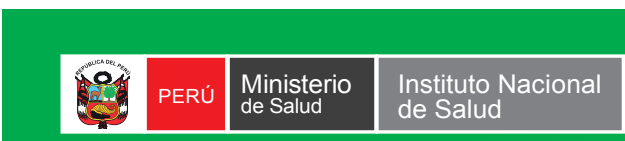

\section{TABLAS PERUANAS DE COMPOSICIÓN DE ALIMENTOS} CENTRO NACIONAL DE ALIMENTACIÓN Y NUTRICIÓN
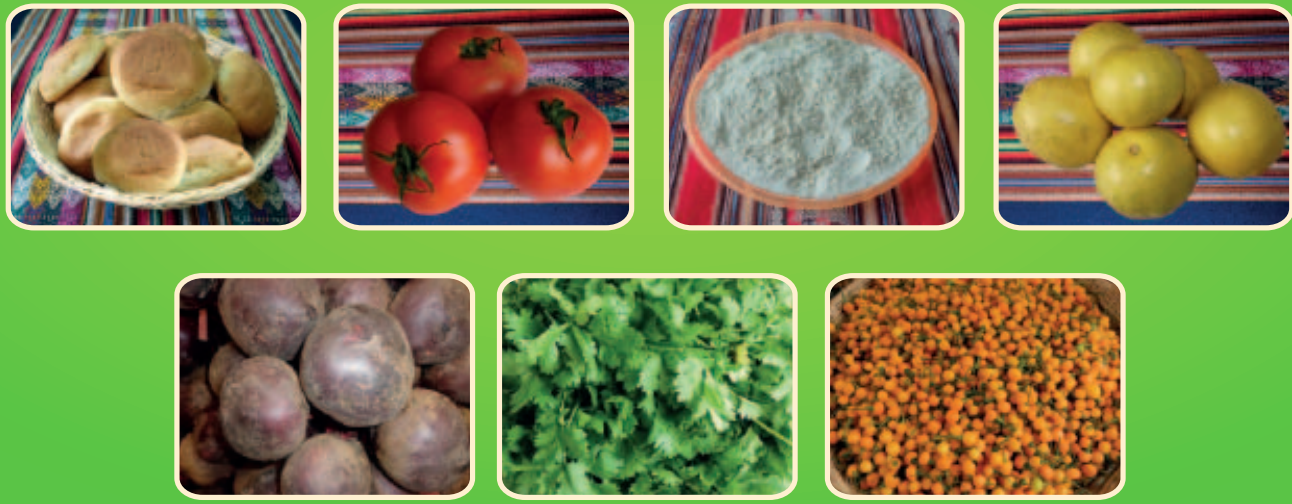

LIMA, 2013

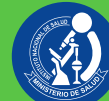

En esta nueva versión se incluye la composición actualizada de 77 nuevos grupos alimenticios, además de los 32 ya disponibles. 